\title{
Health disparities and impact on outcomes in children with primary central nervous system solid tumors
}

\author{
Mary T. Austin, MD, MPH, ${ }^{1-3}$ Emma Hamilton, MD, ${ }^{3}$ Denna Zebda, BS, ${ }^{3}$ Hoang Nguyen, $\mathrm{PhD},{ }^{4}$ \\ Jan M. Eberth, PhD, ${ }^{7}$ Yuchia Chang, DrPH, ${ }^{4}$ Linda S. Elting, DrPH, ${ }^{4}$ and David I. Sandberg, MD $3,5,6$ \\ 1Department of Pediatrics, Children's Cancer Hospital at The University of Texas MD Anderson Cancer Center; Departments \\ of ${ }^{2}$ Surgical Oncology, ${ }^{4} \mathrm{Health}$ Services Research, and ${ }^{6}$ Neurosurgery, The University of Texas MD Anderson Cancer Center; \\ ${ }^{3}$ Department of Pediatric Surgery, University of Texas Medical School at Houston; ${ }^{5}$ Department of Neurosurgery, University of \\ Texas Health Science Center at Houston and Mischer Neuroscience Institute, Houston, Texas; and 'Department of Epidemiology \\ and Biostatistics, University of South Carolina, Columbia, South Carolina
}

\begin{abstract}
OBJECTIVE Health disparities in access to care, early detection, and survival exist among adult patients with cancer. However, there have been few reports assessing how health disparities impact pediatric patients with malignancies. The objective in this study was to examine the impact of racial/ethnic and social factors on disease presentation and outcome for children with primary CNS solid tumors.
\end{abstract}

METHODS The authors examined all children (age $\leq 18$ years) in whom CNS solid tumors were diagnosed and who were enrolled in the Texas Cancer Registry between 1995 and 2009 ( $n=2421)$. Geocoded information was used to calculate the driving distance between a patient's home and the nearest pediatric cancer treatment center. Socioeconomic status (SES) was determined using the Agency for Healthcare Research and Quality formula and 2007-2011 US Census block group data. Logistic regression was used to determine factors associated with advanced-stage disease. Survival probability and hazard ratios were calculated using life table methods and Cox regression.

RESULTS Children with advanced-stage CNS solid tumors were more likely to be $<1$ year old, Hispanic, and in the lowest SES quartile (all $p<0.05$ ). The adjusted odds ratios of presenting with advanced-stage disease were higher in children < 1 year old compared with children > 10 years old (OR $1.71,95 \% \mathrm{Cl} 1.06-2.75$ ), and in Hispanic patients compared with non-Hispanic white patients (OR 1.56, 95\% CI 1.19-2.04). Distance to treatment and SES did not impact disease stage at presentation in the adjusted analysis. Furthermore, 1- and 5-year survival probability were worst in children 1-10 years old, Hispanic patients, non-Hispanic black patients, and those in the lowest SES quartile $(p<0.05)$. In the adjusted survival model, only advanced disease and malignant behavior were predictive of mortality.

CONCLUSIONS Racial/ethnic disparities are associated with advanced-stage disease presentation for children with CNS solid tumors. Disease stage at presentation and tumor behavior are the most important predictors of survival. http://thejns.org/doi/abs/10.3171/2016.5.PEDS15704

KEY WORDS pediatric CNS tumors; race; ethnicity; socioeconomic status; survival; oncology

$\mathrm{B}$ RAIN tumors are the second most common malignancy of childhood and have the highest mortality rate of all childhood cancers (https://nccd.cdc. gov/uscs/). ${ }^{28}$ Disparities in treatment and survival between white and nonwhite patients with cancer are well known in the adult population. Among adult patients with CNS solid tumors, Hispanic and black patients have higher mortality rates, even when controlling for surgical management. ${ }^{8}$ Furthermore, insurance status is also related to survival among adult patients with primary brain tumors. ${ }^{8}$ However, less is known about disparities among the pediatric population with CNS solid tumors. ${ }^{2}$ Several studies,

ABBREVIATIONS $\mathrm{CDC}=$ Centers for Disease Control and Prevention; DSHS $=$ Department of State Health Services; HR = hazard ratio; ICD-O-3 = International Classification of Diseases for Oncology, 3rd Edition; NPCR = National Program of Cancer Registries; OS = overall survival; SEER = Surveillance, Epidemiology and End Results Program; SES = socioeconomic status; TCR = Texas Cancer Registry.

SUBMITTED December 7, 2015. ACCEPTED May 24, 2016.

INCLUDE WHEN CITING Published online August 19, 2016; DOI: 10.3171/2016.5.PEDS15704. 
primarily in children with leukemia and lymphoma, have established that race, socioeconomic status (SES), and access to care can affect outcomes for childhood malignancies. . $^{, 9,30}$

Survival of children after diagnosis of CNS solid tumors is highly dependent on age at diagnosis, the histological subtype, the location of the tumor within the brain, and the treatment regimen. ${ }^{2}$ There have been few studies evaluating racial differences in outcomes of pediatric patients with CNS solid tumors. Population-based studies have demonstrated significant differences in distribution of histological subtypes among races. ${ }^{1}$ However, there are conflicting reports about the impact of race on survival for primary malignant CNS solid tumors in children and adolescents. ${ }^{1,27}$

The objective of our study was to identify health disparities in disease stage at presentation and survival within a large cohort of children with CNS solid tumors whose records were obtained from the Texas Cancer Registry (TCR).

\section{Methods}

We conducted a retrospective study assessing 2421 pediatric patients $\leq 18$ years old with primary CNS solid tumors who presented between January 1, 1995, and December 31,2009 . The study was approved by the institutional review board of the University of Texas MD Anderson Cancer Center and the Texas Department of State Health Services (DSHS). Patient data were obtained from the TCR database (http://www.dshs.state.tx.us/tcr/), a statewide population-based registry that is gold-certified by the North American Association of Central Cancer Registries (http://naaccr.org/certification/certificationlevels.aspx). The TCR is part of the Centers for Disease Control and Prevention (CDC) National Program of Cancer Registries (NPCR), with standardized data collection and quality control protocols. To meet these standards, the TCR has quality control for both internal and external processes to insure the reliability, completeness, consistency, and comparability of TCR data. Data were abstracted on patient demographic data, primary tumor site, stage of disease at diagnosis, first course of treatment, tumor morphology, cause of death, and survival-from medical and laboratory records by trained tumor registrars. Tumor site and histological features were coded according to the WHO criteria in the International Classification of Diseases for Oncology, 3rd Edition (ICD-O-3).$^{10}$ Primary CNS solid tumors were defined by morphology codes 9350 through 9530 (Appendix Table 1).

The TCR data set included patient demographic data (date of birth, sex, race/ethnicity, and home address); date of diagnosis; Surveillance, Epidemiology and End Results Program (SEER)-defined category of stage; date of last follow-up and status; and date of death. Patients were categorized into 3 age groups to reflect infants $(<1$ year old), prepubescent children (1-10 years old), and postpubescent adolescents ( $>10$ years old). Patients were also categorized into 4 race/ethnicity groups (non-Hispanic white, non-Hispanic black, Hispanic, and other). The year of diagnosis was divided into 2 groups: 1995-2002 and
2003-2009, to account for changes in treatment over time. Using SEER-defined categories for disease stage, patients were categorized as having local disease if the tumor was confined to one hemisphere in one part of the brain (infra-/ supratentorial), the meninges, or invading/encroaching on the ventricular system. Regional disease was defined by SEER as tumors that crossed the midline or tentorium, invaded bone, major blood vessels, cranial nerves, or spinal cord. Distant disease was defined as the presence of circulating cells in the CSF, extension to the nasal cavity, nasopharynx, posterior pharynx, or outside the CNS. Patients who had either regional disease or distant metastases were categorized as having advanced disease. Patients with in situ disease were excluded from analysis.

Pediatric cancer treatment centers were identified by 2 methods. First, all hospitals in Texas and bordering states (Arkansas, New Mexico, Louisiana, and Oklahoma) that are members of the Children's Oncology Group (COG, http://www.childrensoncologygroup.org/index.php/locations/) were included. Next, the Texas Hospital Inpatient Discharge Public Use Data File (https://dshs.texas.gov/ thcic/hospitals/Inpatientpudf.shtm) was queried to identify any hospital with $>100$ discharges of children with a primary cancer diagnosis in 2009 . We then mapped and geocoded the location of each treatment center to a street road network available in ArcGIS version 10.0 (Esri). The driving distance between a patient's home address and the nearest pediatric cancer treatment center was calculated and categorized into 0-25 miles, 26-50 miles, and $>50$ miles. These distances were chosen to reflect short $(\leq 25$ miles), intermediate (26-50 miles), and long (> 50 miles) travel distances. We selected $>50$ miles as our long-distance benchmark on the basis of previous work among adult patients with cancer. ${ }^{19,22,25}$

We applied 2007-2011 US Census block group data from the American Community Survey to the following SES index formula, which was developed and validated by the Agency for Healthcare Research and Quality, to calculate the neighborhood SES index score: ${ }^{5}$

$$
\begin{aligned}
& \text { SES Index Score }=50+(-0.07 * \text { crowded })+ \\
& (0.08 * \text { prop } 100)+(-0.10 * \text { pct_poverty })+ \\
& (0.11 * \text { hhinc } 100)+(0.10 * \text { high_educ })+\left(-0.11 * \text { low }_{-}\right. \\
& \text {educ })+(-0.08 * \text { pct_unemp }) .
\end{aligned}
$$

The index components consisted of the following: percentage of households containing $\geq 1$ person per room (crowded); the median home value standardized to range from $0 \%$ to $100 \%$ (prop100); the percentage of persons below the federally defined poverty level (pct_poverty); the median household income standardized to $0-100$ (hhinc100); the percentage of persons age $\geq 25$ years with at least 4 years of college (high_educ); the percentage of persons age $\geq 25$ years with less than a 12th grade education (low_educ); and the percentage of persons age $\geq 16$ years in the labor force who are unemployed and actively seeking work (pct_unemp). The data of the components are available at the block group level from the US Census Bureau American Community Survey. We incorporated each patient's geocoded residential address and the 2010 US census data to calculate a neighborhood SES index 
score, and assigned each patient an SES quartile based on the SES index distribution of the study population. These quartiles are as follows: first quartile, $<25 \%$; second quartile, $25 \%-50 \%$; third quartile, $51 \%-75 \%$; and fourth quartile, $>75 \%$.

Overall survival (OS) was calculated from the date of diagnosis to the date of death from any cause. Patients who were alive at the last follow-up were censored at the last follow-up date. Survival curves were plotted using the Kaplan-Meier method and compared by the log-rank test. One-year and 5-year survival probabilities were estimated using the life table method. Univariate and multivariate logistic regression models were conducted to determine any association between advanced disease-defined as regional or distant metastases-and independent variables including sex, age, race/ethnicity, year of diagnosis, travel distance, SES quartile, and tumor behavior (benign or malignant) at the time of diagnosis. Significant variables in the models were presented with hazard ratio (HR), associated $95 \% \mathrm{CIs}$, and $\mathrm{p}$ values. Multivariate Cox regression analysis was carried out to identify significant predictors of OS. Covariates included in the model were sex, age, race/ethnicity, year of diagnosis, disease stage, travel distance, and SES quartile. Results were reported as HRs with 95\% CIs. All analyses were performed with SAS version 9.3 (SAS Institute). Statistical comparisons were 2 -sided and were considered significant at $\mathrm{p}<0.05$.

\section{Results}

In total, there were 2421 pediatric patients in whom CNS solid tumors were diagnosed between 1995 and 2009 and who were registered in the TCR. The median age was 7 years (range $0-18$ years). Patient demographic data are shown in Table 1 . The majority of patients (83\%) presented with local disease, $11 \%$ with regional metastasis, and $6 \%$ with distant metastasis. The median driving distance from a patient's home to the nearest pediatric cancer treatment center was 19.1 miles (range $<0.2$ to 253.2 miles). The majority of patients lived $\leq 25$ miles from a pediatric cancer treatment center $(60 \%)$. However, $24 \%$ lived $>50$ miles from the nearest pediatric cancer treatment center, and the majority of these counties are classified as either noncore (not part of "core-based" metro- or micropolitan areas) or micropolitan (10,000-49,999 persons) according to the US Census. Malignant tumors were diagnosed in $61 \%$ of patients. The most frequently diagnosed ICD-O-3 codes for the morphological type of tumor are listed in Table 2. A complete list of diagnoses and ICD-O-3 codes is in Appendix Table 1.

The socioeconomic characteristics of the study population are presented in Table 3. The median neighborhood SES index score was 51 (range 31-78). Variability for each component of the SES index is further explored in Table 3. The median poverty level rate was $13 \%$ (range $0 \%-93 \%$ ), and the unemployment rate was $7 \%$ (range $0 \%-45 \%)$.

Results of the univariate and multivariate analyses that evaluated the impact of the covariates on the odds of presenting with advanced-stage disease are shown in Table 4. In the multivariate model, patients $<1$ year old were
TABLE 1. Demographic data of pediatric patients with primary CNS solid tumors

\begin{tabular}{cc}
\hline Characteristic & No. of Patients $(\%)^{*}$ \\
\hline Sex & $1319(54)$ \\
\hline Male & $1102(46)$ \\
\hline Female & $114(5)$ \\
\hline Age group & $1536(63)$ \\
\hline$<1$ yr & $771(32)$ \\
\hline $1-10$ yrs & $1201(50)$ \\
\hline$>10$ yrs & $848(35)$ \\
\hline Race/ethnicity & $270(11)$ \\
\hline Non-Hispanic white & $102(4)$ \\
\hline Hispanic & $1042(43)$ \\
\hline Non-Hispanic black & $1379(57)$ \\
\hline Other & \\
\hline Yr of diagnosis & $2015(83)$ \\
\hline $1995-2002$ & $257(11)$ \\
\hline $2003-2009$ & $149(6)$ \\
\hline Stage of disease & $1445(60)$ \\
\hline Local & $403(17)$ \\
\hline Regional & $573(24)$ \\
\hline Distant & \\
\hline Travel distance & \\
\hline $0-25$ miles & \\
\hline $26-50$ miles & \\
\hline$>50$ miles & \\
\hline
\end{tabular}

* The percentages may not add up to $100 \%$ due to rounding.

more likely to have advanced-stage disease compared with those $>10$ years (OR 1.71, 95\% CI 1.06-2.75). Hispanic patients were $>50 \%$ more likely to have advancedstage disease than non-Hispanic white patients (OR 1.56, 95\% CI 1.19-2.04). Patients with CNS tumors diagnosed between 2003 and 2009 were less likely to have advancedstage disease compared with those diagnosed between

TABLE 2. The ICD-0-3 coding for the most frequent histological type of tumor

\begin{tabular}{llcr}
\hline \multicolumn{1}{c}{ ICD-O-3 } & \multicolumn{1}{c}{ Diagnosis } & Frequency & $\%$ \\
\hline 9421 & Pilocytic astrocytoma & 641 & 26 \\
\hline 9380 & Glioma, malignant & 504 & 21 \\
\hline 9470 & Medulloblastoma NOS & 305 & 13 \\
\hline $9400 \& 9401$ & $\begin{array}{l}\text { Astrocytoma NOS \& anaplastic } \\
\text { astrocytoma }\end{array}$ & 239 & 10 \\
\hline 9391 \& 9392 & $\begin{array}{l}\text { Ependymoma NOS \& anaplastic } \\
\text { ependymoma }\end{array}$ & 207 & 9 \\
\hline 9473 & Primitive neuroectodermal tumor & 105 & 4 \\
\hline 9440 & Glioblastoma NOS & 104 & 4 \\
\hline & Other & 316 & 13 \\
\hline
\end{tabular}

NOS $=$ not otherwise specified. 
TABLE 3. Socioeconomic characteristics of the study population at the block group level

\begin{tabular}{lcrrr}
\hline \multicolumn{1}{c}{ Characteristic } & Mean (SD) & Median & Min & Max \\
\hline SES score & $51.0(7.2)$ & 51 & 31 & 78 \\
\hline $\begin{array}{c}\text { Percent of housing units in } \\
\text { crowded living quarters }\end{array}$ & $5.5(7.4)$ & 3 & 0 & 60 \\
\hline $\begin{array}{c}\text { Ranking based on median house } \\
\text { value; 0\%-100\% }\end{array}$ & $52.6(28.2)$ & 54 & 0 & 100 \\
\hline $\begin{array}{c}\text { Percent living below poverty level } \\
\text { Ranking based on median house- }\end{array}$ & $17.0(15.4)$ & 13 & 0 & 93 \\
\hline $\begin{array}{c}\text { hold income; 0\%-100\% } \\
\text { Percent w/ low education level; no } \\
\text { high school graduation }\end{array}$ & $21.1(17.7)$ & 16 & 0 & 100 \\
\hline $\begin{array}{c}\text { Percent w/ high education level; } \\
\text { college graduation }\end{array}$ & $24.6(20.2)$ & 19 & 0 & 93 \\
\hline \begin{tabular}{c} 
Percent unemployed \\
\hline
\end{tabular} & $7.5(6.2)$ & 7 & 0 & 45 \\
\hline
\end{tabular}

Max = maximum; $\min =$ minimum

1995 and 2002 (OR 0.77, 95\% CI 0.62-0.95). Driving distance to the nearest pediatric cancer treatment center and SES quartile had no significant impact on the stage of disease at presentation.

The Kaplan-Meier survival curves stratified by race/ ethnicity and SES quartile are presented in Figs. 1 and 2 , respectively. Hispanic and non-Hispanic black patients had worse OS compared with non-Hispanic white patients (HR 1.51, 95\% CI 1.29-1.78, and HR 1.51, 95\% CI 1.19-1.90, respectively). Furthermore, patients in the first and second SES quartiles had worse OS compared with patients in the fourth SES quartile (HR 1.39, 95\% CI 1.13-1.70, and HR 1.28, 95\% CI 1.02-1.59, respectively) (Table 5). Race/ethnicity was tightly associated with neighborhood SES (Fig. 3, p < 0.001). Specifically, $50 \%$ of Hispanic and 33\% of non-Hispanic black patients were in the first SES quartile, whereas only $11 \%$ of non-Hispanic white patients were in the first quartile.

In the multivariate Cox regression model, malignant behavior had the most significant impact on survival, whereas race and SES were no longer significant (Table 5; HR 4.64, 95\% CI 3.72-5.74). However, all nonwhite populations were more likely to receive a diagnosis of malignant tumors than non-Hispanic white patients (Fig. 4, $\mathrm{p}<0.001$ ). A subset analysis for patients with malignant tumors only showed that non-Hispanic black patients had significantly worse OS than non-Hispanic white patients (HR 1.30, 95\% CI 1.01-1.68). Regional and distant disease also significantly decreased survival when compared with local disease (HR 1.32, 95\% CI 1.07-1.64, and HR 1.78, 95\% CI 1.40-2.27).

Driving distance to the nearest pediatric cancer treatment center had no impact on OS (Fig. 5 and Table 5).

\section{Discussion}

Neighborhood socioeconomic and racial/ethnic disparities are important factors in disease stage at presentation and survival in children with CNS solid tumors.
TABLE 4. Unadjusted and adjusted odds for presenting with advanced-stage disease*

\begin{tabular}{|c|c|c|c|c|}
\hline \multirow[b]{2}{*}{ Variable } & \multicolumn{2}{|c|}{ Univariate Analysis } & \multicolumn{2}{|c|}{ Multivariate Analysis } \\
\hline & OR & $95 \% \mathrm{Cl}$ & OR & $95 \% \mathrm{Cl}$ \\
\hline \multicolumn{5}{|l|}{ Sex } \\
\hline Male & 0.91 & $0.73-1.12$ & 0.90 & $0.72-1.12$ \\
\hline Female & Reference $†$ & & Reference $†$ & \\
\hline \multicolumn{5}{|l|}{ Age } \\
\hline$<1 \mathrm{yr}$ & 2.03 & $1.27-3.24$ & 1.71 & $1.06-2.75$ \\
\hline $1-10$ yrs & 1.25 & $0.98-1.58$ & 1.12 & $0.87-1.43$ \\
\hline$>10 \mathrm{yrs}$ & Reference† & & Reference† & \\
\hline \multicolumn{5}{|l|}{ Race/ethnicity } \\
\hline $\begin{array}{l}\text { Non- } \\
\text { Hispanic } \\
\text { white }\end{array}$ & Reference $†$ & & Reference $†$ & \\
\hline Hispanic & 1.78 & $1.41-2.24$ & 1.56 & $1.19-2.04$ \\
\hline $\begin{array}{l}\text { Non- } \\
\quad \text { Hispanic } \\
\text { black }\end{array}$ & 1.23 & $0.86-1.77$ & 1.11 & $0.76-1.63$ \\
\hline Other & 0.84 & $0.45-1.57$ & 0.86 & $0.46-1.63$ \\
\hline \multicolumn{5}{|l|}{ Yr of diagnosis } \\
\hline 1995-2002 & Reference $†$ & & Reference† & \\
\hline 2003-2009 & 0.79 & $0.64-0.98$ & 0.77 & $0.62-0.95$ \\
\hline \multicolumn{5}{|l|}{ Travel distance } \\
\hline $0-25$ miles & Reference† & & Reference† & \\
\hline $26-50$ miles & 1.01 & $0.75-1.35$ & 1.15 & $0.85-1.56$ \\
\hline$>50$ miles & 0.86 & $0.66-1.12$ & 0.91 & $0.69-1.21$ \\
\hline \multicolumn{5}{|l|}{ SES quartile } \\
\hline$<25 \%$ & 1.58 & $1.18-2.12$ & 1.19 & $0.85-1.67$ \\
\hline $25 \%-50 \%$ & 1.18 & $0.85-1.63$ & 1.03 & $0.73-1.46$ \\
\hline $51 \%-75 \%$ & 1.03 & $0.75-1.41$ & 0.98 & $0.71-1.36$ \\
\hline$>75 \%$ & Reference $†$ & & Reference $†$ & \\
\hline \multicolumn{5}{|l|}{$\begin{array}{l}\text { Behavior of } \\
\text { lesion }\end{array}$} \\
\hline Benign & Reference† & & Reference† & \\
\hline Malignant & 2.11 & $1.66-2.68$ & 1.99 & $1.55-2.54$ \\
\hline
\end{tabular}

* Advanced disease is defined as either regional or distant disease. Boldface type indicates statistical significance.

$\dagger$ Reference group used in logistic regression models.

Our results support other studies that have evaluated the impact of health disparities on survival in children with cancer. Studies in children with leukemia and lymphoma have indicated that race, SES, and access to care can affect outcomes. ${ }^{3,9,15,23,30}$ In a review article written by Bhatia, ${ }^{2}$ black children with acute myeloid leukemia had a $42 \%$ excess mortality and Hispanic children had a 33\% excess mortality in comparison with their non-Hispanic white counterparts. This difference in mortality is multifactorial and includes a combination of biological factors, SES, and sociocultural factors. In contrast, minimal work has been performed to understand the impact of health disparities on pediatric primary CNS malignancies. This study is the first of its kind to examine the impact of race/ethnicity, 


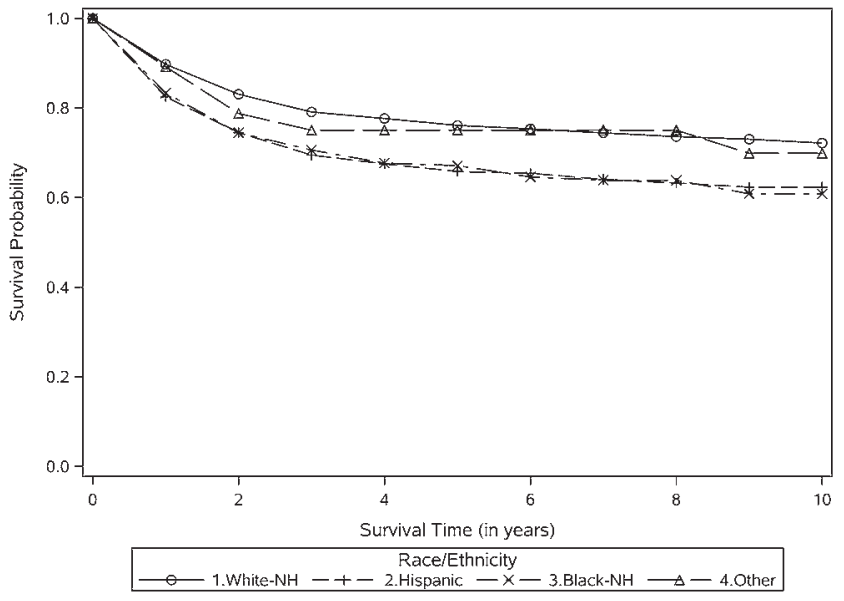

FIG. 1. Graph showing OS curve by race/ethnicity. $\mathrm{NH}=$ non-Hispanic.

SES, and driving distance to the cancer treatment center on disease presentation and OS for children with CNS malignancies.

\section{Race/Ethnicity}

The impact of race/ethnicity on disease presentation and overall survivability has not been studied extensively in pediatric CNS tumors. In a study based on SEER data from 1973 to 1996, OS was similar for Hispanic, black, and Asian patients in whom malignant CNS tumors had been diagnosed, compared with white patients. ${ }^{1}$ However, more recent SEER data from 1992 to 2000 and 2001 to 2007 demonstrated a significant difference in 5-year survival for black pediatric patients with astrocytoma and high-grade glioma compared with white patients. ${ }^{18}$

The results of the current study demonstrated that Hispanic patients were more likely to present with advancedstage disease, and Hispanic and non-Hispanic black patients had decreased OS in comparison with white patients. Previous studies have demonstrated increased prevalence of high-risk disease and decreased OS among black children with neuroblastoma. ${ }^{14}$ Malignant tumor behavior is the most important factor for OS, and Hispanic and nonHispanic black patients were more likely to present with malignant disease compared with non-Hispanic white patients. Moreover, among individuals with malignant tumors, non-Hispanic black patients had worse survival than non-Hispanic white patients.

There may be biological differences between malignant tumors among black and white children. Molecular studies in other tumors such as neuroblastoma have suggested that racial differences in outcomes may potentially be explained by genetic variability in response to drug effectiveness and toxicity, tumor aggression, and resistant or residual disease that relapses or progresses. ${ }^{6,14,16,21}$ Less is known about the molecular genetics of pediatric CNS tumors. Recent advances in understanding the molecular subgroups of medulloblastoma have identified 4 subgroups with variable clinical behavior and outcomes among different age groups. ${ }^{12,31}$ Further understanding of the genetic landscape of CNS tumors among different ra-

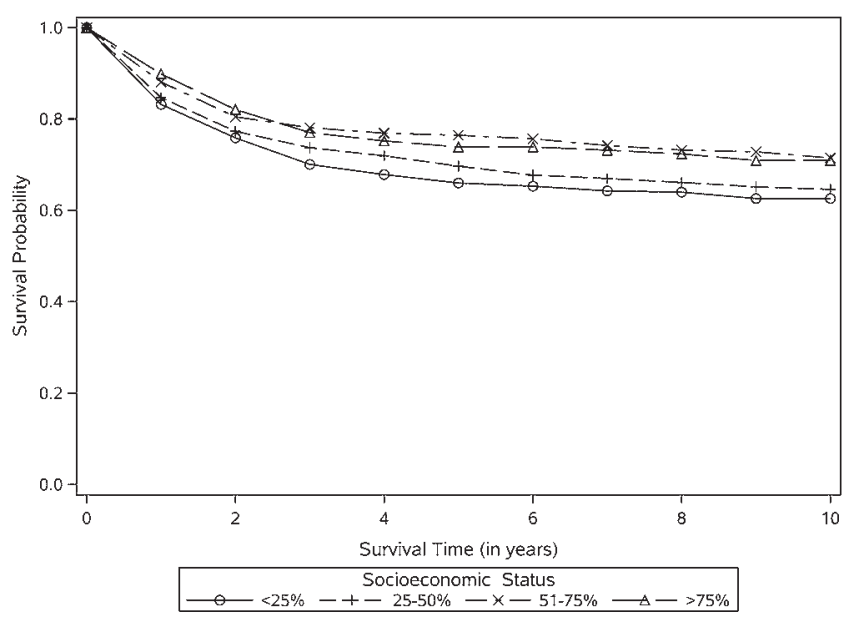

FIG. 2. Graph showing OS curve by SES quartile.

cial/ethnic groups may help explain in part differences in incidence of high-risk disease, advanced-stage disease at presentation, and OS. ${ }^{11}$

Race/ethnicity disparities are multifactorial and may be influenced by social effects. Mukherjee et al. ${ }^{24}$ evaluated factors that influenced access to high-volume centers for neuro-oncological care in the US, and found that Hispanic children were $32 \%$ less likely than their white counterparts to be admitted into high-volume hospitals. It has been shown that high-volume hospitals have improved surgical outcomes with fewer complications, indirectly impacting OS. ${ }^{24}$ Furthermore, studies using the SEER database have demonstrated that Hispanic and non-Hispanic black children had lower 5-year survival rates compared with non-Hispanic white children for many cancer types, including CNS solid tumors. ${ }^{13,20}$ However, single-institution studies have failed to demonstrate a racial disparity in survival from pediatric cancer. ${ }^{27}$ Single-center studies may suffer more from greater selection bias than population-based studies, especially for those institutions that provide protocol-driven care with no direct charge to patients. These findings suggest that socioeconomic factors may play a significant role in observed differences in survival. ${ }^{27}$

\section{Socioeconomic Status}

Access to quality health care, timely diagnosis, and ability to maintain compliance with recommended treatment regimens are closely associated with SES, ethnicity, and survival. ${ }^{29}$ Population studies among adult patients with CNS tumors have demonstrated socioeconomic disparities in surgical management, access to high-volume centers, and increased in-hospital mortality after resection. ${ }^{4,8,17,32}$ Less is known about SES disparities in access to care among pediatric patients with CNS tumors. A study from the US between 1988 and 2005 revealed that Hispanic patients and those with a lower SES had less access to high-volume neuro-oncological care compared with non-Hispanic white patients and those with a higher SES. ${ }^{24}$ However, survival outcomes were not evaluated. An earlier study from Wales between 1971 and 1990 


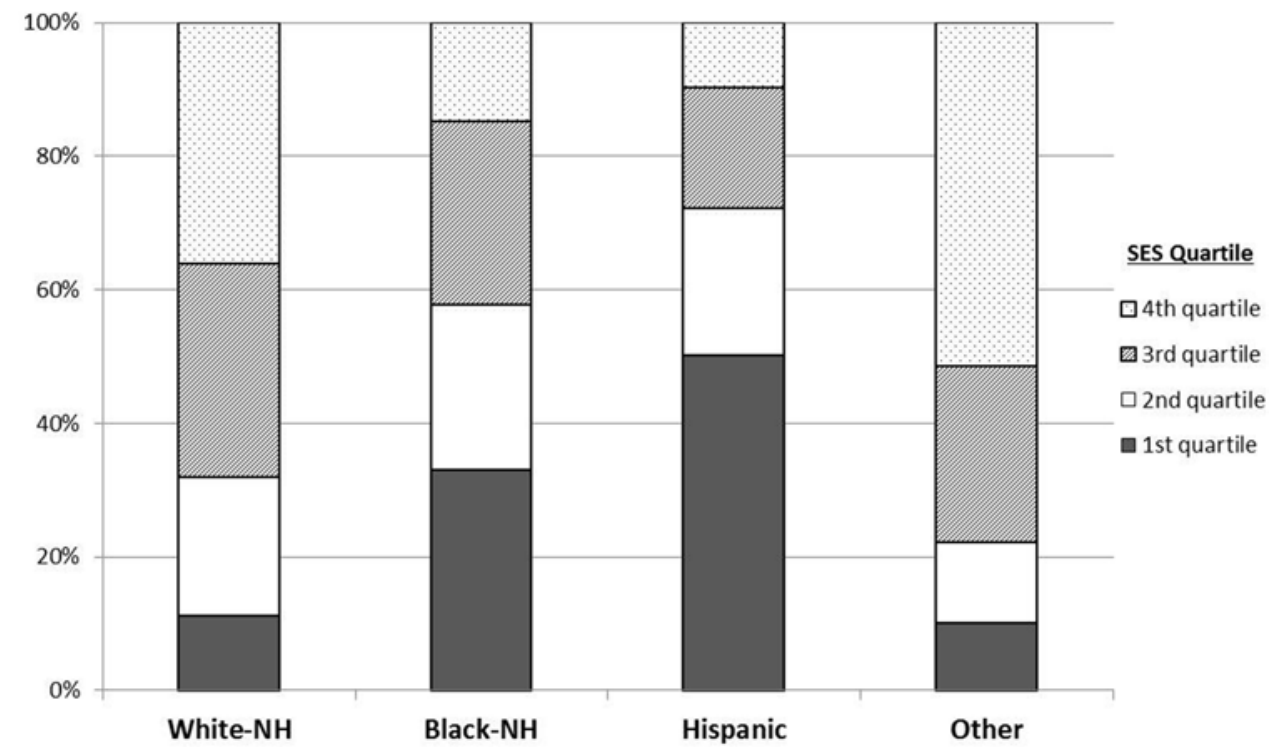

FIG. 3. Bar graph showing the distribution of SES quartiles within each racial/ethnic group. There were significant differences in SES by race. $\chi^{2}=509, p<0.0001$.

found that SES did not affect survival in pediatric patients with CNS solid tumors. ${ }^{33}$

Our results demonstrated that children in the first SES quartile were more likely to present with advanced-stage disease. However, when controlling for race and other sociodemographic characteristics, SES was no longer associated with advanced-stage disease, whereas race/ethnicity remained predictive of presentation with advanced-stage disease. Furthermore, patients in the first and second SES quartiles had decreased OS compared with patients in the fourth SES quartile. However, when controlling for other factors, SES was no longer a significant predictor of survival. In our study, race/ethnicity was closely associated with SES. More than half of the Hispanic and non-Hispanic black children were within the first and second SES quartiles, compared with only $32 \%$ of non-Hispanic white children. Because race/ethnicity and SES are multifaceted and so closely intertwined, it is difficult to determine in our study whether potential genetic variability or SES disparities among different racial/ethnic groups contribute more to presentation of advanced disease and the decrease in OS.

\section{Travel Distance for Treatment}

We found no relationship between driving distance to the nearest pediatric cancer treatment center and stage of disease presentation or OS. Our study is the first to evaluate travel distance to a pediatric cancer center on survival outcomes of pediatric patients with CNS solid tumors. The SEER data have demonstrated that pediatric patients with neuroblastoma living in nonmetropolitan counties have a higher mortality rate than those living in metropolitan counties..$^{15}$ In our study, the majority of patients lived $\leq 25$ miles from a pediatric cancer treatment center, whereas < $25 \%$ of patients lived $>50$ miles from the nearest center. The limited number of patients living far from a pediatric cancer center may have hindered our ability to detect a difference. Furthermore, it is important to note that in our study travel distance was defined as the nearest potential treatment facility and not the actual distance traveled to the facility used by the patient. The goal was to evaluate the impact of access to care on disease presentation and overall survivability. This approach is based on the underlying assumption that access improves with geographic proximity. ${ }^{25}$ Future studies are needed to evaluate the impact of the distance actually traveled by patients (i.e., realized access) on outcome measures such as stage of presentation, adherence to and completion of therapy, follow-up surveillance, and OS.

\section{Year of Diagnosis}

In this series, individuals in whom a CNS tumor was diagnosed between 2003 and 2009 were more likely to present with localized disease than individuals whose tumor was diagnosed between 1995 and 2002. This finding remained significant after controlling for other sociodemographic variables and tumor behavior. This finding may reflect increased access to care and earlier diagnosis. There may also have been changes in the distribution and incidence of tumor histological types during these periods., ${ }^{7,26}$ However, year of diagnosis was not associated with OS.

\section{Limitations and Strengths}

The data for the study were obtained from the TCR database. The current study has several strengths. The TCR has high-quality data that are closely monitored for accuracy in each patient. Although the TCR has a large Hispanic population, this is the fastest-growing population in the US, and our results are generalizable to the US population. The large Hispanic population in this database is also useful in elucidating racial/ethnic disparities in outcomes of pediatric patients with CNS solid tumors. 
TABLE 5. Cox regression to determine independent predictors for OS*

\begin{tabular}{|c|c|c|c|c|c|c|}
\hline \multirow[b]{2}{*}{ Variable } & \multicolumn{3}{|c|}{ Univariate Analysis } & \multicolumn{3}{|c|}{ Multivariate Analysis } \\
\hline & $\mathrm{HR}$ & $\mathrm{Cl}$ & p Value & $H R$ & $\mathrm{Cl}$ & $p$ Value \\
\hline \multicolumn{7}{|l|}{ Sex } \\
\hline Male & 1.03 & $0.89-1.19$ & 0.7072 & 0.93 & $0.80-1.08$ & 0.3206 \\
\hline Female & Reference† & & & eference & & \\
\hline \multicolumn{7}{|l|}{ Age } \\
\hline$<1 \mathrm{yr}$ & 1.67 & $1.22-2.30$ & 0.0016 & 1.21 & $0.87-1.69$ & 0.2602 \\
\hline $1-10$ yrs & 1.23 & $1.05-1.44$ & 0.0123 & 1.09 & $0.92-1.29$ & 0.9722 \\
\hline$>10 \mathrm{yrs}$ & Reference† & & & eference & & \\
\hline \multicolumn{7}{|l|}{ Race/ethnicity } \\
\hline Non-Hispanic white & Reference $†$ & & & eference & & \\
\hline Hispanic & 1.51 & $1.29-1.78$ & $<0.0001$ & 1.14 & $0.95-1.37$ & 0.1658 \\
\hline Non-Hispanic black & 1.51 & $1.19-1.90$ & 0.0006 & 1.26 & $0.99-1.61$ & 0.0613 \\
\hline Other & 1.09 & $0.73-1.65$ & 0.6669 & 0.93 & $0.61-1.44$ & 0.7538 \\
\hline \multicolumn{7}{|l|}{ Yr of diagnosis } \\
\hline 1995-2002 & Reference $†$ & & & eference & & \\
\hline $2003-2009$ & 0.90 & $0.77-1.04$ & 0.1577 & 0.87 & $0.75-1.02$ & 0.0782 \\
\hline \multicolumn{7}{|l|}{ Behavior of lesion } \\
\hline Benign & Reference $†$ & & & eference & & \\
\hline Malignant & 5.08 & $4.10-6.29$ & $<0.0001$ & 4.64 & $3.72-5.74$ & $<0.0001$ \\
\hline \multicolumn{7}{|l|}{ Stage of disease } \\
\hline Local & Reference $†$ & & & eference & & \\
\hline Regional & 1.58 & $1.28-1.96$ & $<0.0001$ & 1.32 & $1.07-1.64$ & 0.0109 \\
\hline Distant & 2.40 & $1.89-3.05$ & $<0.0001$ & 1.78 & $1.40-2.27$ & $<0.0001$ \\
\hline \multicolumn{7}{|l|}{ Travel distance } \\
\hline $0-25$ miles & Reference $†$ & & & eference & & \\
\hline $26-50$ miles & 0.89 & $0.72-1.10$ & 0.2744 & 0.97 & $0.78-1.20$ & 0.7769 \\
\hline$>50$ miles & 0.87 & $0.73-1.05$ & 0.1401 & 0.91 & $0.76-1.11$ & 0.3504 \\
\hline \multicolumn{7}{|l|}{ SES quartile } \\
\hline$<25 \%$ & 1.39 & $1.13-1.70$ & 0.0019 & 1.13 & $0.90-1.43$ & 0.3056 \\
\hline $25 \%-50 \%$ & 1.28 & $1.02-1.59$ & 0.0311 & 1.17 & $0.93-1.48$ & 0.1824 \\
\hline $51 \%-75 \%$ & 0.99 & $0.78-1.22$ & 0.8407 & 0.97 & $0.77-1.22$ & 0.7919 \\
\hline$>75 \%$ & Reference $†$ & & & eference & & \\
\hline
\end{tabular}

* Boldface type indicates statistical significance.

$\dagger$ Reference group used in multivariate Cox regression models.

The study has several limitations, including those inherent in all retrospective investigations. We chose to define advanced disease as regional or metastatic disease. Prior to 2010, the SEER definition of regional CNS tumors included tumors that crossed the midline. This definition may inadvertently label patients with localized midline tumors that involve both the right and left hemisphere as having regional disease. Furthermore, the TCR did not begin recording insurance status until 2007. Due to the small number of patients whose insurance status was available, we were not able to evaluate the influence of insurance status on outcomes. Insurance status is closely associated with a number of factors, including race/ethnicity and SES, and may be a potentially important variable on disease stage at presentation and OS. In this study, we wanted to evaluate the patient's access to care and distance to the nearest pediatric treatment center rather than the actual distance

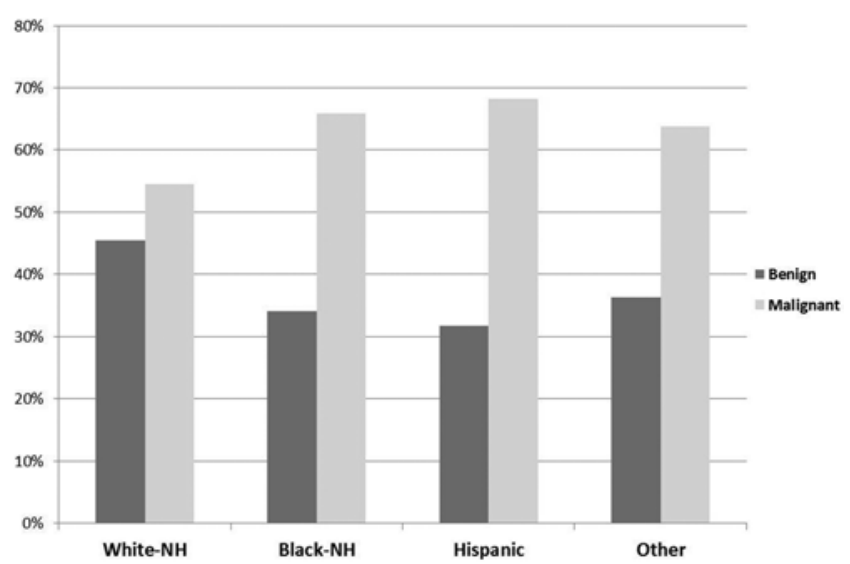

FIG. 4. Bar graph showing the distribution of race/ethnicity among pediatric patients with CNS solid tumors. There were significant differences in tumor behavior by race. $\chi^{2}=43, p<0.0001$. 


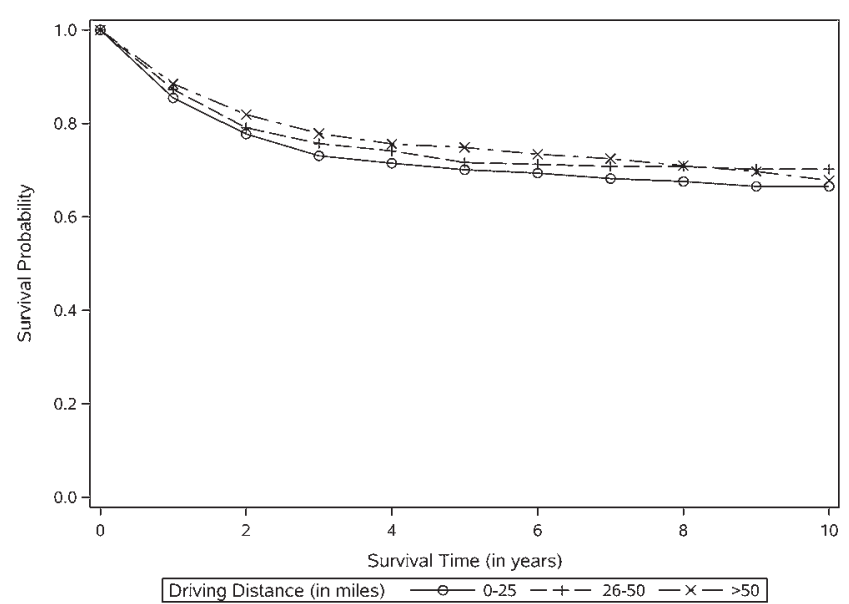

FIG. 5. Graph showing OS curve by driving distance to the nearest pediatric cancer treatment center.

traveled to the treatment center used by the patient. Also, we were unable to account for patients who moved closer to treatment facilities postdiagnosis. Furthermore, we were not able to include hospital-specific factors, such as high- or low-volume center, that may have impacted OS. ${ }^{24}$

\section{Conclusions}

Racial/ethnic disparities are associated with advancedstage disease presentation for children with CNS solid tumors. Furthermore, disease stage at presentation and tumor behavior are the most important predictors of survival. The underlying causes for these disparities are multifactorial and complex. Future studies are needed to elucidate the specific causes of these disparities to develop interventions to improve outcomes among pediatric patients with CNS solid tumors.

\section{Acknowledgments}

This work was supported by the Cancer Prevention Research Institute of Texas (CPRIT), Grant No. RP101207. Dr. Jan Eberth was the recipient of a cancer prevention fellowship supported by the National Cancer Institute (R25T CA5730; Shine Chang, PhD, Principal Investigator) and the National Institutes of Health (MD Anderson Cancer Center Support Grant No. CA016672) during the course of the study. The collection of cancer incident data used in this study was supported by the Texas DSHS and CPRIT, as part of the statewide cancer reporting program, and the CDC's NPCR Cooperative Agreement \#5U58/DP000824-05. Its contents are solely the responsibility of the authors and do not necessarily represent the official views of the DSHS, CPRIT, or CDC.

We thank Melanie A. Williams, PhD, Branch Manager, TCR, Texas DSHS, Austin, Texas, for her invaluable feedback and assistance with this project.

\section{References}

1. Barnholtz-Sloan JS, Severson RK, Stanton B, Hamre M, Sloan AE: Pediatric brain tumors in non-Hispanics, Hispanics, African Americans and Asians: differences in survival after diagnosis. Cancer Causes Control 16:587-592, 2005

2. Bhatia S: Disparities in cancer outcomes: lessons learned from children with cancer. Pediatr Blood Cancer 56:9941002,2011
3. Bhatia S: Influence of race and socioeconomic status on outcome of children treated for childhood acute lymphoblastic leukemia. Curr Opin Pediatr 16:9-14, 2004

4. Birkmeyer NJO, Gu N, Baser O, Morris AM, Birkmeyer JD: Socioeconomic status and surgical mortality in the elderly. Med Care 46:893-899, 2008

5. Bonito AJ, Bann C, Eicheldinger C, Carpenter L: Creation of New Race-Ethnicity Codes and Socioeconomic Status (SES) Indicators for Medicare Beneficiaries. Final Report. Rockville, MD: Agency for Healthcare Research and Quality, 2008

6. Capasso M, Devoto M, Hou C, Asgharzadeh S, Glessner JT, Attiyeh EF, et al: Common variations in BARD1 influence susceptibility to high-risk neuroblastoma. Nat Genet 41:718723,2009

7. Claus EB, Black PM: Survival rates and patterns of care for patients diagnosed with supratentorial low-grade gliomas: data from the SEER program, 1973-2001. Cancer 106:13581363,2006

8. Curry WT Jr, Barker FG II: Racial, ethnic and socioeconomic disparities in the treatment of brain tumors. J Neurooncol 93:25-39, 2009

9. Darmawikarta D, Pole JD, Gupta S, Nathan PC, Greenberg $\mathrm{M}$ : The association between socioeconomic status and survival among children with Hodgkin and non-Hodgkin lymphomas in a universal health care system. Pediatr Blood Cancer 60:1171-1177, 2013

10. Fritz A, Percy C, Jack A, Shanmugaratnam K, Sobin L, Parkin DM, et al (eds): International Classification of Diseases for Oncology (ICD-O), ed 3. Geneva: World Health Organization, 2013

11. Gabriel A, Batey J, Capogreco J, Kimball D, Walters A, Tubbs RS, et al: Adult brain cancer in the U.S. black population: a Surveillance, Epidemiology, and End Results (SEER) analysis of incidence, survival, and trends. Med Sci Monit 20:1510-1517, 2014

12. Gajjar A, Bowers DC, Karajannis MA, Leary S, Witt H, Gottardo NG: Pediatric brain tumors: Innovative genomic information is transforming the diagnostic and clinical landscape. J Clin Oncol 33:2986-2998, 2015

13. Gupta S, Wilejto M, Pole JD, Guttmann A, Sung L: Low socioeconomic status is associated with worse survival in children with cancer: a systematic review. PLoS One 9:e89482, 2014

14. Henderson TO, Bhatia S, Pinto N, London WB, McGrady P, Crotty C, et al: Racial and ethnic disparities in risk and survival in children with neuroblastoma: a Children's Oncology Group study. J Clin Oncol 29:76-82, 2011

15. Hsieh MH, Meng MV, Walsh TJ, Matthay KK, Baskin LS: Increasing incidence of neuroblastoma and potentially higher associated mortality of children from nonmetropolitan areas: analysis of the surveillance, epidemiology, and end results database. J Pediatr Hematol Oncol 31:942-946, 2009

16. Huang RS, Duan S, Kistner EO, Hartford CM, Dolan ME: Genetic variants associated with carboplatin-induced cytotoxicity in cell lines derived from Africans. Mol Cancer Ther 7:3038-3046, 2008

17. Iwamoto FM, Reiner AS, Panageas KS, Elkin EB, Abrey LE: Patterns of care in elderly glioblastoma patients. Ann Neurol 64:628-634, 2008

18. Johnson DR, O'Neill BP: Glioblastoma survival in the United States before and during the temozolomide era. J Neurooncol 107:359-364, 2012

19. Lin CC, Bruinooge SS, Kirkwood MK, Hershman DL, Jemal A, Guadagnolo BA, et al: Association between geographic access to cancer care and receipt of radiation therapy for rectal cancer. Int J Radiat Oncol 94:719-728, 2016

20. Linabery AM, Ross JA: Childhood and adolescent cancer survival in the US by race and ethnicity for the diagnostic period 1975-1999. Cancer 113:2575-2596, 2008 
21. Maris JM, Mosse YP, Bradfield JP, Hou C, Monni S, Scott $\mathrm{RH}$, et al: Chromosome 6p22 locus associated with clinically aggressive neuroblastoma. N Engl J Med 358:2585-2593, 2008

22. Massarweh NN, Chiang YJ, Xing Y, Chang GJ, Haynes AB, You YN, et al: Association between travel distance and metastatic disease at diagnosis among patients with colon cancer. J Clin Oncol 32:942-948, 2014

23. Mostert S, Sitaresmi MN, Gundy CM, Sutaryo, Veerman AJ: Influence of socioeconomic status on childhood acute lymphoblastic leukemia treatment in Indonesia. Pediatrics 118:e1600-e1606, 2006

24. Mukherjee D, Kosztowski T, Zaidi HA, Jallo G, Carson BS, Chang DC, et al: Disparities in access to pediatric neurooncological surgery in the United States. Pediatrics 124:e688e696, 2009

25. Onega T, Duell EJ, Shi X, Wang D, Demidenko E, Goodman D: Geographic access to cancer care in the U.S. Cancer 112:909-918, 2008

26. Patel S, Bhatnagar A, Wear C, Osiro S, Gabriel A, Kimball $\mathrm{D}$, et al: Are pediatric brain tumors on the rise in the USA? Significant incidence and survival findings from the SEER database analysis. Childs Nerv Syst 30:147-154, 2014

27. Pui CH, Boyett JM, Hancock ML, Pratt CB, Meyer WH, Crist WM: Outcome of treatment for childhood cancer in black as compared with white children. The St Jude Children's Research Hospital experience, 1962 through 1992. JAMA 273:633-637, 1995

28. Siegel DA, King J, Tai E, Buchanan N, Ajani UA, Li J: Cancer incidence rates and trends among children and adolescents in the United States, 2001-2009. Pediatrics 134:e945e955, 2014

29. Smedley B, Stith A, Nelson A: Unequal Treatment: Confronting Racial and Ethnic Disparities in Health Care. Washington, DC: National Academies Press, 2003

30. Smith EC, Ziogas A, Anton-Culver H: Association between insurance and socioeconomic status and risk of advanced stage Hodgkin lymphoma in adolescents and young adults. Cancer 118:6179-6187, 2012

31. Taylor MD, Northcott PA, Korshunov A, Remke M, Cho YJ, Clifford SC, et al: Molecular subgroups of medulloblastoma: the current consensus. Acta Neuropathol 123:465-472, 2012
32. Trinh VT, Davies JM, Berger MS: Surgery for primary supratentorial brain tumors in the United States, 2000-2009: effect of provider and hospital caseload on complication rates. J Neurosurg 122:280-296, 2015

33. Tseng JH, Tseng MY: Survival analysis of children with primary malignant brain tumors in England and Wales: a population-based study. Pediatr Neurosurg 42:67-73, 2006

\section{Disclosures}

Dr. Elting received salary support for this work from the Comparative Effectiveness Research on Cancer in Texas (CERCIT), Grant No. RP140020, which was funded by the CPRIT.

\section{Author Contributions}

Conception and design: Austin, Hamilton, Elting, Sandberg. Acquisition of data: Austin, Zebda, Nguyen, Eberth, Chang, Elting, Sandberg. Analysis and interpretation of data: all authors. Drafting the article: Austin, Zebda, Chang. Critically revising the article: Austin, Hamilton, Nguyen, Eberth, Elting, Sandberg. Reviewed submitted version of manuscript: all authors. Approved the final version of the manuscript on behalf of all authors: Austin. Statistical analysis: Nguyen, Chang. Administrative/technical/ material support: Chang. Study supervision: Sandberg.

\section{Supplemental Information Online-Only Content}

Supplemental material is available with the online version of the article.

Appendix Table 1. http://thejns.org/doi/suppl/10.3171/2016.5. PEDS15704.

\section{Previous Presentations}

Portions of this work were given as an oral presentation at the annual meeting of the AANS, held in San Francisco, California, on April 5-9, 2014.

\section{Correspondence}

Mary T. Austin, Department of Surgical Oncology, The University of Texas MD Anderson Cancer Center, 1400 Pressler, Unit 1406, Houston, TX 77030-1439. email: maustin@mdanderson.org. 